\title{
Application of fungal fluorescent staining in oral candidiasis: diagnostic analysis of 228 specimens
}

\author{
Yilin Yao ${ }^{1,2,3}$, Linjun Shi ${ }^{1,2,3}$, Chunye Zhang ${ }^{2,3,4}$, Hong Sun ${ }^{5}$ and Lan $\mathrm{Wu}^{1,2,3^{*}}$ (D)
}

\begin{abstract}
Background: Several conventional methods, including fungal culture and periodic acid-Schiff (PAS) reagent staining, have been used to diagnose oral candidiasis. The aim of this study was to evaluate the efficacy of a novel method, fungal fluorescent staining, in relation to conventional protocols in the diagnosis of oral candidiasis.

Methods: We collected 106 oral swabs and 122 oral biopsy tissues from patients highly suspected with oral candidiasis. We applied fungal culture and periodic acid-Schiff reagent staining as the gold standard diagnostic tools. The efficacy of these methods in determining the presence of Candida was compared with that of fluorescent staining.

Results: In the majority of specimens subjected to fluorescent staining, fungal organisms were distinguished by blue fluorescence surrounding their tubular or annular shapes. The sensitivity, specificity, Youden index, positive predictive value and negative predictive value of the fluorescent staining method were 82.7, 93.5, 76.7, 96.8 and $69.1 \%$ in oral swabs and 90.0, 92.9, 82.9, 96.0 and $82.9 \%$ in oral biopsy tissues, respectively.

Conclusions: Fungal fluorescent staining represents a rapid method for detection of Candida, supporting its potential utility as an effective early diagnostic tool for oral candidiasis.
\end{abstract}

Keywords: Fungal fluorescent staining, Oral candidiasis, Fungal culture, Periodic acid-Schiff reagent staining

\section{Background}

Candida, an opportunistic pathogen, is a commensal and harmless organism under normal conditions that becomes invasive; pathogenic pseudohyphae overbreed locally and cause oral candidiasis upon disruption of the balance of flora or debilitationof the host $[1,2]$. The disease is most commonly caused by overgrowth of Candida albicans in the mouth [3]. Diagnosis of oral candidiasis is generally based on thorough analysis of medical history and physical examination, and confirmed by examining oral swabs or oral biopsy tissue samples. The conventional methods adopted for diagnosis in these two sample types are fungal culture [4-6] and PAS staining $[4,7,8]$. However, both techniques are time-consuming and lead to false-negative results in $5-15 \%$ cases [9], highlighting the necessity for

\footnotetext{
*Correspondence: teana_wu@sina.com

'Department of Oral Mucosal Diseases, Shanghai Ninth People's Hospital,

College of Stomatology, Shanghai Jiao Tong University School of Medicine, Shanghai, China

${ }^{2}$ National Clinical Research Center for Oral Diseases, Shanghai, China

Full list of author information is available at the end of the article
}

more rapid and sensitive methods. Fungal fluorescent staining is currently under investigation as a potential means to effectively detect Candida infection. While several studies have documented the successful application of fluorescent staining for fungal detection in nail specimens [10-12], limited reports are available on diagnosis using oral samples. This study was designed to compare fungal fluorescent staining with conventional methods, with a view to establishing an optimal technique for accurate and rapid diagnosis of oral candidiasis.

\section{Results}

Clinical specimens and participants

The features of 228 specimens with suspected oral candidiasis (Figs. 1a and 2a) are summarized in Table 1. In the first phase, 106 oral swabs were obtained from 41 males and 65 females aged $25-78$ years (average age, 47.6 years). Overall, 66 males and 56 females participated in the second phase of the study. Participant ages ranged from 27 to 82 years (median age, 55.9 years). The majority of biopsy 

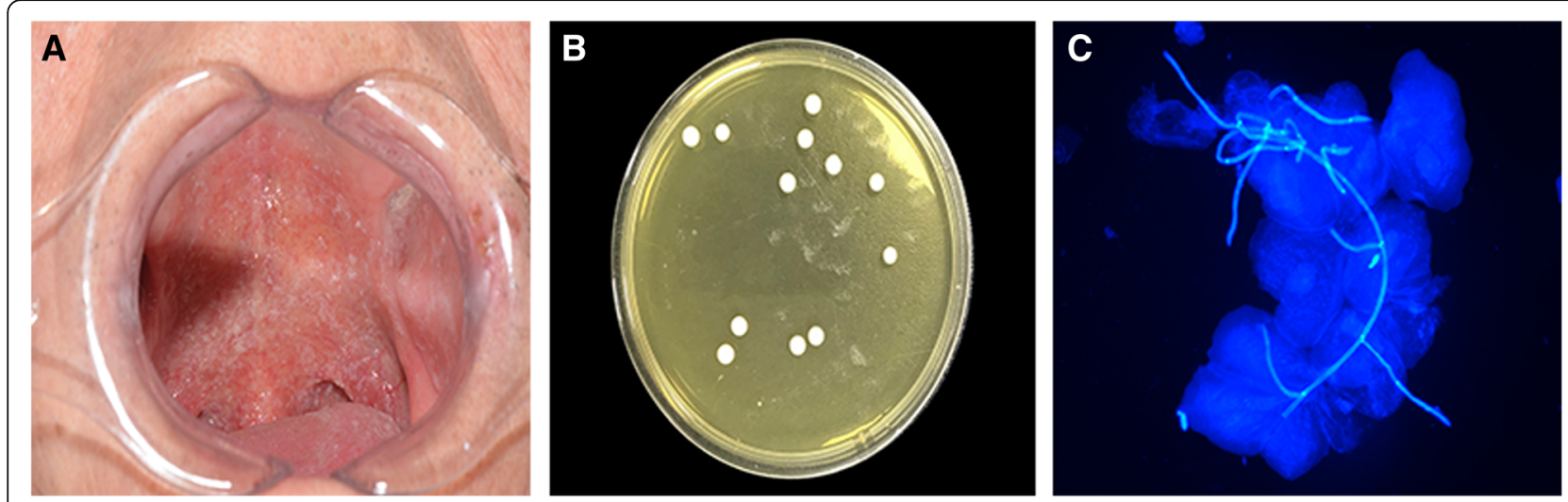

Fig. 1 a Clinical manifestation of highly suspected oral candidiasis. b Presence of Candida colonies in the culture-positive case. c Fungal hyphae surrounded by strong blue fluorescence under fluorescence microscopy $(\times 40)$

tissues were histologically diagnosed as oral leukoplakia (59.8\%), followed by oral lichen planus (15.6\%), oral squamous cell carcinoma (14.8\%) and oral mucositis (9.3\%).

\section{Fungal detection in oral swabs}

In culture-positive cases, unique fungal colonies of Candida (Fig. 1b) were found on Sabouraud's Dextrose Agar (SDA) that were white to cream in color, smooth, glabrous, with a yeast-like appearance and smell. Upon growth on Chromogenic Candida Agar (CCA), colonies of C. albicans are characteristically green, C. glabrata purple, and C. tropicalis bluish-grey.

In fluorescence-positive cases, hyphae (Fig. 1c) and spores appeared bright blue against a black background, and fungal morphology was easily distinguishable in oral swabs.

Among the 106 oral swabs, fungal culture was positive in 75 samples (70.7\%), among which 13 turned out negative with the fluorescent staining technique, and negative in 31 samples (29.3\%), among which 2 were positive with fluorescent staining. These data are presented in Table 2. Among the culture-positive samples, $C$. albicans was detected in 58 cases, C. glabrata in 10 cases and C. tropicalis in 7 cases. Importantly, the fluorescent staining technique required an average time of only $10 \mathrm{~min}$ while fungal culture was conducted over 6 days.

\section{Fungal detection in oral tissues}

In PAS-positive cases, Candida hyphae invaded the epithelium at right angles to the surface (Fig. 2b). The fungal appearance of fluorescent staining in tissue specimens was similar to that in oral swabs but not as easy to distinguish (Fig. 2c).

In total, 80 specimens (65.6\%) were PAS-positive, among which 8 displayed negative fluorescent staining, and 42 (34.4\%) were PAS-negative, among which 3 were detected as positive using fluorescent staining. These data are presented in Table 3. The fluorescent technique required $1 \mathrm{~min}$ to generate specimens and $10-15 \mathrm{~min}$ to read samples, compared to a longer period of $2 \mathrm{~h}$ for specimen generation and $10-20 \mathrm{~min}$ for reading the results with PAS staining.
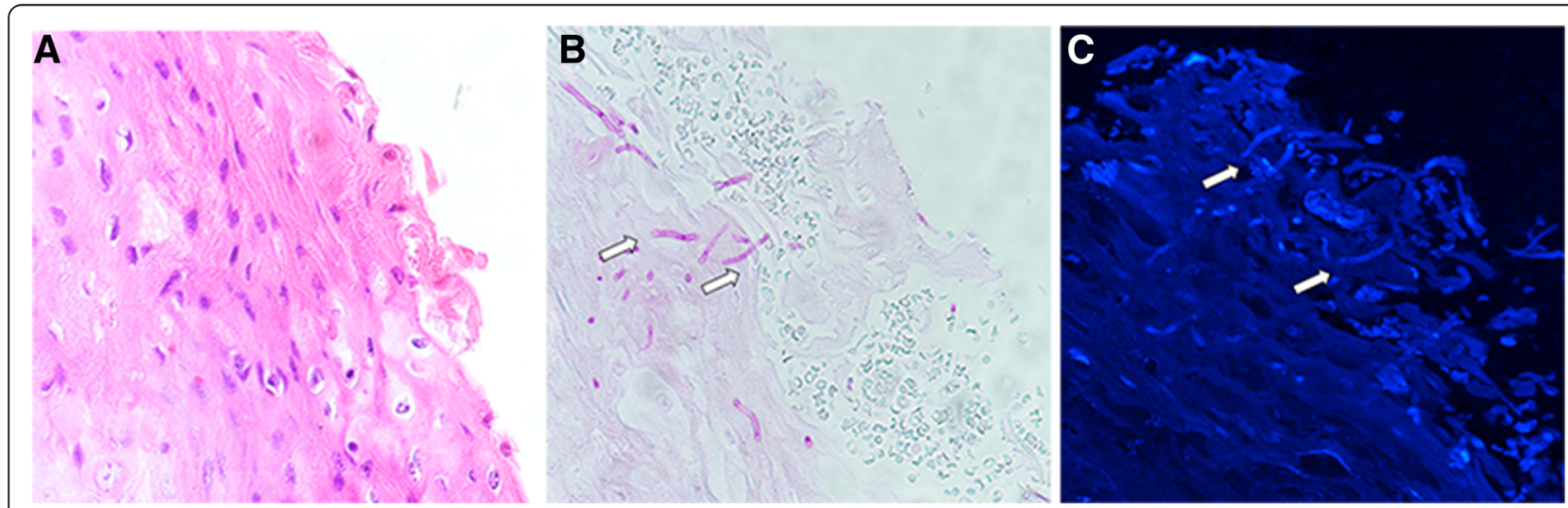

Fig. 2 a A case of highly suspected oral candidiasis via HE staining under light microscopy ( $\times 40$ ). b Fungal organism (white arrows) on PAS of same specimen $(\times 40)$. c Fungal organisms from the same section as B distinguishable via fluorescence microscopy as tubular or tubulo-annular structures (white arrows) with a thin rim of bright blue fluorescence $(\times 40)$ 
Table 1 Baseline characteristics of 228 specimens

\begin{tabular}{lll}
\hline Feature & Swabs & Biopsy tissues \\
\hline Total & 106 & 122 \\
Age(years) & & \\
$\quad$ Mean & 47.6 & 55.9 \\
$\quad$ Range & $25-78$ & $27-82$ \\
Sex & & \\
$\quad$ Males & $41(38.7 \%)$ & $66(45.9 \%)$ \\
Females & $65(61.3 \%)$ & $56(54.1 \%)$ \\
Histology & & \\
$\quad$ Oral squamous cell carcinoma & $/$ & $18(14.8 \%)$ \\
$\quad$ Oral leukoplakia & $/$ & $73(59.8 \%)$ \\
Oral lichen planus & $19(15.6 \%)$ \\
Oral mucositis & $/$ & $12(9.8 \%)$ \\
\end{tabular}

Sensitivity, specificity, Youden index, and negative and positive predictive values

For determination of each parameter, conventional methods (culture-/PAS-positive) were used as the gold standard. For oral swabs and oral biopsy tissues, the sensitivity of fluorescent staining was 82.7 and $90.0 \%$, and specificity was 93.5 and $92.9 \%$, respectively. The positive predictive values were 96.8 and $96.0 \%$, negative predictive values were 69.1 and $82.9 \%$, and Youden indexes were 76.2 and $82.9 \%$, respectively (> 70\%). All three diagnostic methods for oral candidiasis are compared in Table 4.

\section{Discussion}

The diagnosis of oral candidiasis is fundamentally clinical [1]. Microbiological techniques or culture are often used to identify hyphae or spores for confirmation of diagnosis [13]. Timely and efficient laboratory diagnosis is essential to facilitate the implementation of effective antifungal treatments. In this study, we evaluated the utility of fluorescent staining in the diagnosis of oral candidiasis, with a view to establishing a clinically appropriate rapid and efficient Candida detection technique. The efficacy of fungal fluorescent staining as a diagnostic tool was compared with conventional fungal culture and PAS staining methods.

Fungal fluorescent staining is a novel diagnostic method for oral candidiasis. The fluorescent antibody in the

Table 2 The results of Candida detection in oral swabs

\begin{tabular}{llll}
\hline Technique & \multicolumn{2}{l}{ Culture } & Total \\
\cline { 2 - 3 } & Positive & Negative & \\
\hline FLO & 62 & 2 & 64 \\
Positive & 13 & 29 & 42 \\
Negative & 75 & 31 & 106 \\
Total & & &
\end{tabular}

FLO fluorescent staining
Table 3 The results of Candida detection in oral biopsy tissues

\begin{tabular}{llll}
\hline Technique & PAS & Total \\
\cline { 2 - 3 } & Positive & Negative & \\
\hline FLO & 72 & 3 & 75 \\
Positive & 8 & 39 & 47 \\
Negative & 80 & 42 & 122 \\
Total & &
\end{tabular}

Calcofluor white (CFW) fluorescent dye specifically binds to glucan and chitin layers unique to the fungal cell wall [14]. Thus, fluorescein, which emit blue fluorescence under illumination, is indirectly labeled upon specific binding to the fungal cell wall. And then clear visualization of the profile of the fungus was observed. In a diagnostic study by Okamoto MR [5] using culture as the gold standard, fluorescent staining had $84 \%$ sensitivity and $100 \%$ specificity, resulting in a positive predictive value of $100 \%$ and negative predictive value of $65 \%$. Kirani KR [15] conducted a similar investigation, which disclosed $100 \%$ sensitivity, 93.3\% specificity, a negative predictive value of $100 \%$ and a positive predictive value of $85.7 \%$. The sensitivity of fluorescent staining was high, supporting its value in the diagnosis of Candida infection. Using fungal culture as the gold standard in this study, the sensitivity, specificity, Youden index, positive predictive value and negative predictive value obtained with the fluorescent staining method were 82.7, 93.5, 76.7, 96.8 and $69.1 \%$, respectively, further validating the suitability of this method. It is important to note that fluorescent staining requires only $10 \mathrm{~min}$, compared with 6 days of fungal culture, further providing a guarantee of early diagnosis. Experienced staff found fluorescent staining preparations easy and rapid and the appearance of hyphae or spores bright and distinguishable using fluorescence microscopy [16]. Overall, the fluorescent technique was safe, quick and reliable, all of which are important considerations in a busy diagnostic laboratory.

One of the earliest descriptions of fungal fluorescence in tissue sections was reported by Graham in 1983 [17], who recommended fluorescent staining as a supplementary detection technique. Subsequently, Elston [18] reported the application of fluorescent staining in nail samples, $66 \%$ of which were positive. Additionally, moderate to strong

Table 4 Sensitivity, specificity, positive and negative predictive value and Youden index for all diagnostic methods

\begin{tabular}{lll}
\hline Specimens & Oral swabs & Oral biopsy tissues \\
\hline Sensitivity & $82.7 \%$ & $90.0 \%$ \\
Specificity & $93.5 \%$ & $92.9 \%$ \\
Positive predictive value & $96.8 \%$ & $96.0 \%$ \\
Negative predictive value & $69.1 \%$ & $82.9 \%$ \\
Youden index & $76.7 \%$ & $82.9 \%$ \\
\hline
\end{tabular}


fluorescence was detected in $74 \%$ oral squamous cell carcinoma tissues by Jahanshahi [19]. Data from both studies were consistent with our results, showing strong fluorescence in $61.5 \%$ of specimens. In this investigation, detection of fluorescence was related to the density of fungi in specimens and background fluorescence of keratin, which could be confused with fungal fluorescence. However, a similar problem exists with PAS staining [19]. Moreover, distinguishing species based on morphology is a challenge with both methods. Accurate identification of fungal species may be achieved in conjunction with other techniques, such as immunohistochemistry [20], in situ hybridization [21] and polymerase chain reaction [22]. In our tissue analysis, fungal fluorescent staining showed high sensitivity (90.0\%), specificity (92.9\%) and Youden index (82.9\%), validating the conclusions of Graham [17]. Fluorescent staining of oral biopsy tissues has several distinct advantages to PAS staining. Firstly, results are obtained almost instantaneously. Secondly, this method is a more economical option than PAS. Thirdly, tissue sections that have been stained with fluorescence dyes can be reused.

Above all, our findings support the utility of fungal fluorescent staining in early diagnosis of oral candidiasis. We conclude that fluorescent staining serves as a rapid, simple and convenient screening tool for detection of fungi in oral specimens.

\section{Conclusions}

Accuracy and speed are the highly desirable goals for fungal detection. The time required for fluorescent staining is shorter than that for the corresponding gold standard in oral swabs and oral biopsy tissues. In both specimen types, fungal fluorescent staining shows high sensitivity, specificity and Youden index supporting its value as a potential new method for early detection of Candida.

\section{Methods}

\section{Clinical specimens}

The study was divided into two phases. In the first phase, 106 patients with clinically suspected oral candidiasis were enrolled. Two oral swabs were obtained at the same time from lesions of the patients. Written informed consent based on the guidelines and agreements of the institutional ethical committee was obtained. All specimens were collected by the same experienced mycologist. In the second phase, 122 hematoxylin-eosin (HE)-stained tissue specimens of oral mucosal diseases with suspected Candida colonization between 2010 and 2018 were retrieved from the archive and two serial sections obtained from each specimen.

\section{Fungal detection in oral swabs}

In the first phase, two oral swabs were examined via culture and fungal fluorescent staining. For fluorescent staining, the swab was applied onto a glass slide. A $50 \mu \mathrm{L}$ drop of CFW fluorescent dye (Liming Biotech Co., Ltd., Nanjing, China) was placed on the slide for $30 \mathrm{~s}$ and excess dye gently removed with blotting paper. The slide was covered with a cover glass and examined under a fluorescence microscope (Nikon Ni-U, Japan) using blue light excitation $(300-400 \mathrm{~nm}$ for emission wavelength with excitation at around $355 \mathrm{~nm}$ ).

A sample culture was performed in parallel on SDA (Hope Biotech Co., Ltd., Qingdao, China) at $25^{\circ} \mathrm{C}$ for $5-7$ days. The rate and texture of growth, surface color, and color on reverse and diffusible pigments were determined. Culture-positive strains were subsequently streaked on CCA (Chromagar Microbiology, Paris, France) and further incubated at $37^{\circ} \mathrm{C}$ for $24 \mathrm{~h}$. Candida species were identified by colony color according to the manufacturer's chart.

\section{Fungal detection in oral biopsy tissues}

In the second phase, two sections were used for diagnosis via PAS and fungal fluorescent staining. In the fluorescent staining procedure, after dewaxing the sample,the same operation procedure was performed as before.

In terms of PAS staining, sections were incubated in $0.1 \%$ periodic acid for $5-8 \mathrm{~min}$ after dewaxing, washed in running tap water for 2-3 min and immersed in Schiff'sreagent for $10-20 \mathrm{~min}$. Subsequently, sections were washed in tap water for $10 \mathrm{~min}$, counterstained with hematoxylin for $2 \mathrm{~min}$, differentiated in acidic ethanol for $2-3 \mathrm{~s}$ and washed in tap water for $10 \mathrm{~min}$. Finally, sections were dehydrated with ethanol, cleared in xylene and mounted with neutral balsam (Sinopharm Chemical Reagent Co., Ltd., Shanghai, China).

Prior to the study, all participants were trained in the relevant Standard OperationProcedures (SOPs). Diagnosis of a sample was assessed by two experts in oral pathology or laboratory medicine who reached an inter-observer agreement. The total time taken for each experiment was recorded.

\section{Statistical analysis}

Sensitivity, specificity, Youden index, and negative and positive predictive value were calculated using fungal culture as the gold standard for the first phase and PAS staining as the gold standard for the second phase.

\section{Abbreviations}

CCA: Chromogenic Candida Agar; CFW: Calcofluor white; HE: Hematoxylin-eosin; PAS: Periodic acid-Schif; SDA: Sabouraud's Dextrose Agar

\section{Acknowledgements}

Not applicable.

Funding

The design of this work was supported by grants from National Key R\&D Program of China (2017YFC0840100, 2017YFC0840110). Data collection was granted by

Clinical Research Plan of SHDC and Shanghai's top priority clinical medicine center 
(2017ZZ01011). The funders played no part in data analysis, interpretation of data and writing of the manuscript.

\section{Availability of data and materials}

All data generated or analyzed during current study are available from the corresponding author on reasonable request.

\section{Authors' contributions}

LW planned and executed the experiments. YY and LS collected the clinical specimens. $\mathrm{CZ}$ and $\mathrm{HS}$ detected fungal in oral biopsy tissues and oral swabs respectively. $Y$ Y analyzed the data. All authors read and approved the final manuscript.

\section{Ethics approval and consent to participate}

This study was approved by Institutional Ethical Committee, Ninth People's Hospital, Shanghai Jiao Tong University School of Medicine (SH9H-2019-T35-1). Written informed consent was obtained from each participant after the investigator had explained the nature of the study.

\section{Consent for publication}

Not applicable.

\section{Competing interests}

The authors declare that they have no competing interests.

\section{Publisher's Note}

Springer Nature remains neutral with regard to jurisdictional claims in published maps and institutional affiliations.

\section{Author details}

'Department of Oral Mucosal Diseases, Shanghai Ninth People's Hospital, College of Stomatology, Shanghai Jiao Tong University School of Medicine, Shanghai, China. ${ }^{2}$ National Clinical Research Center for Oral Diseases, Shanghai, China. ${ }^{3}$ Shanghai Key Laboratory of Stomatology \& Shanghai Research Institute of Stomatology, Shanghai, China. ${ }^{4}$ Department of Oral Pathology, Shanghai Ninth People's Hospital, College of Stomatology, Shanghai Jiao Tong University School of Medicine, Shanghai, China. ${ }^{5}$ Department of Laboratory Medicine, Shanghai Ninth People's Hospital, Shanghai Jiao Tong University School of Medicine, Shanghai, China.

Received: 29 November 2018 Accepted: 26 April 2019

Published online: 14 May 2019

\section{References}

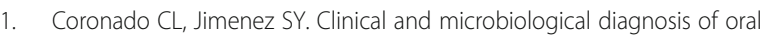
candidiasis. J Clin Exp Dent. 2013;5(5):279-86.

2. Reichart PA, Samaranayake LP, Philipsen HP. Pathology and clinical correlates in oral candidiasis and its variants: a review. Oral Dis. 2000;6(2):85-91.

3. Millsop JW, Fazel N. Oral candidiasis. Clin Dermatol. 2016;34(4):487-94.

4. Cuenca EM, Verweij PE, Arendrup MC, Arikan-Akdagli S, Bille J, Donnelly JP, Jensen HE, Lass-Floerl C, Richardson MD, Akova M, et al. ESCMID* guideline for the diagnosis and management of Candida diseases 2012: diagnostic procedures. Clin Microbiol Infect. 2012;18:9-18.

5. Okamoto MR, Kamoi M, Yamachika S, Tsurumoto A, Imamura T, Yamamoto K, Kadomatsu S, Saito I, Maeda N, Nakagawa Y. Efficacy of Fungiflora Y staining for the diagnosis of oral erythematous candidiasis. Gerodontology. 2013;30(3):220-5.

6. Ilkit M, Guzel AB. The epidemiology, pathogenesis, and diagnosis of vulvovaginal candidosis: a mycological perspective. Crit Rev Microbiol. 2011; 37(3):250-61.

7. Sitheeque MAM, Samaranayake LP. Chronic hyperplastic candidosis/ candidiasis (Candidal leukoplakia). Crit Rev Oral Biol Med. 2003;14(4):253-67.

8. Jeelani S, Ahmed QM, Lanker AM, Hassan I, Jeelani N, Fazili T. Histopathological examination of nail clippings using PAS staining (HPE-PAS): gold standard in diagnosis of onychomycosis. Mycoses. 2015;58(1):27-32.

9. Bao F, Fan Y, Sun L, Yu Y, Wang Z, Pan Q, Yu C, Liu H, Zhang F. Comparison of fungal fluorescent staining and ITS rDNA PCR-based sequencing with conventional methods for the diagnosis of onychomycosis. J Eur Acad Dermatol Venereol. 2018;32(6):1017-21.
10. Gupta AK, Versteeg SG, Shear NH. Onychomycosis in the 21st century: an update on diagnosis, epidemiology, and treatment. J Cutan Med Surg. 2017; 21(6):525-39.

11. Idriss $M$ H, Khalil A, Elston D. The diagnostic value of fungal fluorescence in onychomycosis. J Cutan Pathol. 2013;40(4):385-90.

12. Ghannoum M, Mukherjee $P$, Isham N, Markinson B, Rosso JD, Leal L. Examining the importance of laboratory and diagnostic testing when treating and diagnosing onychomycosis. Int J Dermatol. 2018;57(2):131-8.

13. Williams DW, Lewis MA. Isolation and identification of Candida from the oral cavity. Oral Dis. 2000;6(1):3-11.

14. Chaffin WL, Lopez-Ribot JL, Casanova M, Gozalbo D, Martinez JP. Cell wall and secreted proteins of Candida albicans: identification, function, and expression. Microbiol Mol Biol Rev. 1998;62(1):130.

15. Kirani KR, Chandrika VS. Efficacy of in-house fluorescent stain for fungus. Indian J Pathol Microbiol. 2017:60(1):57-60.

16. Hamer EC, Moore CB, Denning DW. Comparison of two fluorescent whiteners, Calcofluor and Blankophor, for the detection of fungal elements in clinical specimens in the diagnostic laboratory. Clin Microbiol Infect. 2006; 12(2):181-4.

17. Graham AR. Fungal autofluorescence with ultraviolet illumination. Am J Clin Pathol. 1983;79(2):231-4.

18. Elston DM. Fluorescence of fungi in superficial and deep fungal infections. BMC Microbiol. 2001;1:1-4.

19. Jahanshahi G, Shirani S. Detection of Candida albicans in oral squamous cell carcinoma by fluorescence staining technique. Dent Res J (Isfahan). 2015; 12(2):115-20.

20. Jensen HE, Schonheyder HC, Hotchi M, Kaufman L. Diagnosis of systemic mycoses by specific immunohistochemical tests: review article. APMIS. 1996; 104(4):241-58.

21. Lischewski A, Amann RI, Harmsen D, Merkert H, Hacker J, Morschhaeuser J. Specific detection of Candida albicans and Candida tropicalis by fluorescent in situ hybridization with an $18 \mathrm{~S}$ rRNA-targeted oligonucleotide probe. Microbiology. 1996;142(10):2731-40.

22. Munoz-Cadavid C, Rudd S, Zaki SR, Patel M, Moser SA, Brandt ME, Gomez $\mathrm{BL}$. Improving molecular detection of fungal DNA in formalin-fixed paraffinembedded tissues: comparison of five tissue DNA extraction methods using Panfungal PCR. J Clin Microbiol. 2010;48(6):2147-53.

Ready to submit your research? Choose BMC and benefit from:

- fast, convenient online submission

- thorough peer review by experienced researchers in your field

- rapid publication on acceptance

- support for research data, including large and complex data types

- gold Open Access which fosters wider collaboration and increased citations

- maximum visibility for your research: over $100 \mathrm{M}$ website views per year

At $\mathrm{BMC}$, research is always in progress.

Learn more biomedcentral.com/submissions 